\title{
Liprin- $\alpha 4$ as a Possible New Therapeutic Target for Pancreatic Cancer
}

\author{
AKIO YAMASAKI ${ }^{1}$, KAZUNORI NAKAYAMA ${ }^{1}$, AKIRA IMAIZUMI ${ }^{2}$, MAKOTO KAWAMOTO $^{1}$, \\ AKIKO FUJIMURA ${ }^{1}$, YASUHIRO OYAMA ${ }^{1}$, SHUNTARO NAGAI ${ }^{3}$, KOSUKE YANAI $^{1}$ and HIDEYA ONISHI ${ }^{1}$ \\ Departments of ${ }^{1}$ Cancer Therapy and Research, ${ }^{3}$ Surgery and Oncology, \\ Graduate School of Medical Sciences, Kyushu University, Fukuoka, Japan; \\ ${ }^{2}$ Shukoukai Inc., Tokyo, Japan
}

\begin{abstract}
Background/Aim: In pancreatic cancer, where the microenvironment is extremely hypoxic, analyzing signal transduction under hypoxia is thought to be significantly important. By investigating microarray analysis of pancreatic cancer cells cultured under both normoxia and hypoxia, we found that the expression of leukocyte common antigen-related (LAR)-interacting protein (liprin)- $\alpha 4$ was extremely increased under hypoxia compared to under normoxia. Materials and Methods : In the present study, the biological significance of liprin- $\alpha 4$ in pancreatic cancer was investigated and whether liprin- $\alpha 4$ has potential as a therapeutic target for pancreatic cancer was estimated. Results: Suppression of liprin- $\alpha 4$ reduced proliferation of pancreatic cancer cells both in vitro and in vivo. Inhibition of liprin- $\alpha 4$ also reduced invasiveness through the suppression of endothelial-mesenchymal transition. Stimulation by liprin- $\alpha 4$ was through phosphoinositide 3-kinase and mitogen-activated protein kinase signaling pathways. Conclusion: Liprin-a4 plays a pivotal role in inducing malignant phenotypes such as increased proliferation and invasion in pancreatic cancer, and that liprin- $\alpha 4$ could be a new effective therapeutic target for pancreatic cancer.
\end{abstract}

Pancreatic cancer remains one of the deadliest types of cancer, associated with an overall survival rate of $<5 \%$ (1). One reason for this may be that few patients can undergo curative operations because the disease has already advanced at the time of diagnosis. In addition, there are still few effective chemotherapy agents for the treatment of refractory pancreatic cancer. The development of novel therapeutic strategies is urgently required.

Correspondence to: Hideya Onishi, Department of Cancer Therapy and Research, Graduate School of Medical Sciences, Kyushu University, 3-1-1 Maidashi, Higashi-ku, Fukuoka 812-8582, Japan. Tel: +81926426220, Fax: +81926426221, e-mail: ohnishi@ surg1.med.kyushu-u.ac.jp

Key Words: Liprin- $\alpha 4$, pancreatic cancer cells.
The microenvironment of pancreatic cancer is extremely hypoxic (2). The contributions of particular signaling pathways or molecules, which are activated under hypoxia but not under normoxia, can be considered a cause of pancreatic cancer intractability. Therefore, analysis of the molecules and signaling network activated under hypoxic conditions is required to develop a new effective therapeutic strategy for pancreatic cancer. Many researchers have shown that hypoxiainducible factor (HIF)-1 $\alpha$ is an important transcriptional factor in cancer under hypoxia $(3,4)$. However, it is reported that many other signaling pathways are also activated under hypoxia, independently of HIF-1 $\alpha$. For example, we reported that Hedgehog $(\mathrm{Hh})$ signaling, recombination signal-binding protein for immunoglobulin-kappa-J region (RBPJ), and mastermind-like 3 (MAML3) are activated under hypoxia independently of HIF-1 $\alpha$ and they are involved in the induction of malignant phenotypes of cancer $(5,6)$.

Recently, it was shown that leukocyte common antigenrelated (LAR)-interacting protein (liprin)- $\alpha 4$ is up-regulated under hypoxic conditions and that it is directly regulated by HIF- $1 \alpha$ in renal cell carcinoma (7). Liprin- $\alpha$ belongs to the LAR family of receptor protein tyrosine phosphatase $(8,9)$. Of liprin- $\alpha$ families, liprin- $\alpha 1$ is well researched and has been shown to be required for the congregation of neuronal synapses (10) and cell migration (11). Liprin- $\alpha 1$ also contributes to invasiveness of cancer cells $(12,13)$. However, the biological significance of liprin- $\alpha 4$ in pancreatic ductal adenocarcinoma (PDAC) cells under hypoxia remains unclear.

In the present study, in order to develop an effective therapeutic strategy for refractory pancreatic cancer, we investigated whether liprin- $\alpha 4$ has potential as a new therapeutic target molecule for pancreatic cancer.

\section{Materials and Methods}

Cell culture and reagents. Three human PDAC cell lines (ASPC-1, SUIT-2 and Panc-1: American Type Culture Collection, Mannassas, VA, USA) were maintained in RPMI-1640 medium (Nacalai Tesque, Kyoto, Japan) supplemented with $10 \%$ fetal calf serum 
(FCS; Life Technologies Grand Island, NY, USA) and antibiotics (100 units/ml of penicillin and $100 \mu \mathrm{g} / \mathrm{ml}$ of streptomycin). For normoxic conditions, cells were cultured in $5 \% \mathrm{CO}_{2}$ and $95 \%$ air. For hypoxic conditions, cells were cultured in $1 \% \mathrm{O}_{2}, 5 \% \mathrm{CO}_{2}$, and $94 \% \mathrm{~N}_{2}$ using a multigas incubator (Sanyo, Tokyo, Japan). Cell numbers were counted under light microscopy.

Microarray analysis. Methodology of DNA microarray we used was described previously (15). According to the manufacturer's protocol, the cDNA was amplified and labeled using Low Input Quick Amp Labeling (Agilent Technologies, Santa Clara, CA, USA), and hybridized using SurePrint G3 Human Gene Expression Microarray $8 \times 60 \mathrm{k}$ v2 (Agilent). All hybridized microarray slides were scanned using an Agilent scanner. Relative hybridization intensities and background hybridization values were calculated using Agilent Feature Extraction Software (ver. 9.5.1.1). Intensity based Z-scores and ratios (nonlog-scale fold-change) were calculated from the normalized signal intensities of each probe for comparison between cells (ASPC-1 and SUIT-2) cultured under normoxia, and cells cultured under hypoxia.

RNA interference. siRNA for liprin- $\alpha 4$ (ON-TARGETplus SMART pool, L-190474) and negative control siRNA (ON-TARGETplus si CONTROL non-targeting pool, D-001810) were purchased from Dharmacon RNA Technologies (Chicago, IL, USA). Cells $\left(2 \times 10^{5}\right.$ cells/well) seeded in 6-well plates were transfected with $100 \mathrm{nM}$ siRNA using Lipofectamine RNAiMAX Reagent (Thermo Fisher Scientific, Waltham, MA USA) according to the manufacturer's instructions. Cells were assayed at 2 days after transfection.

Matrigel invasion assay. The invasiveness of pancreatic cancer cells was assessed based on the invasion of cells through Matrigel-coated transwell inserts as described previously (14). In brief, the upper surface of a filter (pore size, $8.0 \mu \mathrm{m}$; BD Biosciences, Heidelberg, Germany) was coated with basement membrane Matrigel (BD Biosciences). Cells were suspended in RPMI-1640 with $10 \%$ FCS then $8 \times 10^{4}$ cells were added to the upper chamber and incubated for $16 \mathrm{~h}$. After incubation, the filter was fixed and stained with DiffQuick reagent (International Reagents, Kobe, Japan). All cells that had migrated from the upper to the lower side of the filter were counted under a light microscope (BX50; Olympus, Tokyo, Japan) at a magnification of $\times 100$. Tumor cell invasiveness testing was carried out in triplicate wells.

Western blotting. Whole cell extraction was performed with M-PER Reagents (Pierce Biotechnology, Rockford, IL, USA) according to the manufacturer's instructions. Protein samples $(50 \mu \mathrm{g})$ were separated by electrophoresis on an sodium dodecyl sulfate polyacrylamide gel and transferred to Protran nitrocellulose membranes (Whatman GmbH, Dassel, Germany). The proteintransferred membranes were incubated overnight at $4^{\circ} \mathrm{C}$ with primary antibodies for matrix metalloproteinase (MMP) 2 (1:200, sc-10736; Santa Cruz Biotechnology, Dallas, TX, USA), MMP9 (1:200, sc-6840; Santa Cruz Biotechnology), E-cadherin (1:200, sc7870; Santa Cruz Biotechnology), vimentin (1:200, sc-6260; Santa Cruz Biotechnology), SNAI2 (SLUG) (1:200, sc-15391; Santa Cruz Biotechnology), SNAI1 (SNAIL 1)(1:200, sc-10433, Santa Cruz Biotechnology), TWIST (1:200, sc-15393; Santa Cruz Biotechnology), p- extracellular signal-regulated kinase (ERK)1/2 (1:200; Cell Signaling Technology, Danvers, MA, USA), ERK1/2
Table I. Expression of liprin- $\alpha 4$ in pancreatic ductal adenocarcinoma cells under hypoxia compared with that under normoxia in microarray analysis.

\begin{tabular}{llllll}
\hline & \multicolumn{4}{c}{ Cell line } \\
\cline { 2 - 3 } \cline { 5 - 6 } & \multicolumn{2}{c}{ ASPC-1 } & & \multicolumn{2}{c}{ SUIT-2 } \\
\cline { 2 - 3 } \cline { 5 - 6 } Z-score & Ratio & & Z-score & Ratio \\
\hline Liprin- $\alpha 4$ & 9.205912 & 311.4109 & & 6.740904 & 117.9236 \\
\hline
\end{tabular}

(1:200; Cell signaling Technology), p-AKT1/2/3 (1:200, sc-101629; Santa Cruz Biotechnology), or AKT1/2/3 (1:200, sc-8312; Santa Cruz Biotechnology). Peroxidase-linked secondary antibodies (Amersham Biosciences, Piscataway, NJ, USA) were subsequently added and the membranes were further incubated for $1 \mathrm{~h}$ at room temperature. The antibody for $\alpha$-tubulin (1:1,000; Sigma-Aldich, St. Louis, MO, USA) was used as protein loading control.

Mouse xenograft model. Five-week-old female athymic nude mice (BALB/c nu/nu) were purchased from Charles River Laboratories Japan (Kanagawa, Japan) and acclimated for one week. All experimental procedures were approved by the Animal Care and Use Committee of Kyushu University (permit number: A27-324-0). All mice were housed and maintained in a specific pathogen-free animal facility at Kyushu University, and all efforts were made to minimize the number of animals used and their suffering. All experiments were performed in strict accordance with the Guidelines for Proper Conduct of Animal Experiments (Science Council of Japan). SUIT-2 cells transfected with liprin- $\alpha 4$ siRNA or control siRNA were subcutaneously implanted into the flank of nude mice $\left(1 \times 10^{6}\right.$ cells in Matrigel per mouse; $n=3$ in each treatment group). Tumor size was measured twice a week until 5 weeks, volume was calculated as follows: $\mathrm{A} \times \mathrm{B}^{2} \times 0.5$, where $\mathrm{A}$ is the longest diameter and $\mathrm{B}$ is the smaller of the two perpendicular diameters of the tumor, and then mice were sacrificed.

Fluorescent immunohistochemistry. Slides from tumors formed in mice were deparaffinized with xylene, and rehydrated with alcohol; antigen retrieval was achieved by microwaving in Target Retrieval Solution (pH 6.0, DAKO, Japan) for $10 \mathrm{~min}$. The sections were rinsed with phosphate-buffered saline and blocked using Blocking One Histo (Nacalai Tesque) for $10 \mathrm{~min}$ at room temperature. The sections were incubated with anti-Ki67 (sc-15402; Santa Cruz Biotechnology), antivascular endothelial growth factor (VEGF) (sc-152; Santa Cruz Biotechnology), anti-pAKT (sc-101629; Santa Cruz Biotechnology) and anti-pERK1/2 (Cell Signaling Technology), antibodies overnight at $4^{\circ} \mathrm{C}$. Primary antibodies were then visualized by incubating slides with Alexa Fluor 594-conjugated goat anti-rabbit (1:1,000; Invitrogen) for $1 \mathrm{~h}$ at $37^{\circ} \mathrm{C}$, respectively. After incubation with secondary antibodies, sections were rinsed with phosphate-buffered saline three times. Slides were counterstained with 4',6-diamidino-2phenylindole (DAPI; Sigma Aldrich, St. Louis, MI, USA) and then mounted by VectaShield (Vector Laboratories, Burlingame, CA, USA). The samples were qualitatively examined under fluorescence microscopy (Carl Zeiss, Tokyo, Japan). 

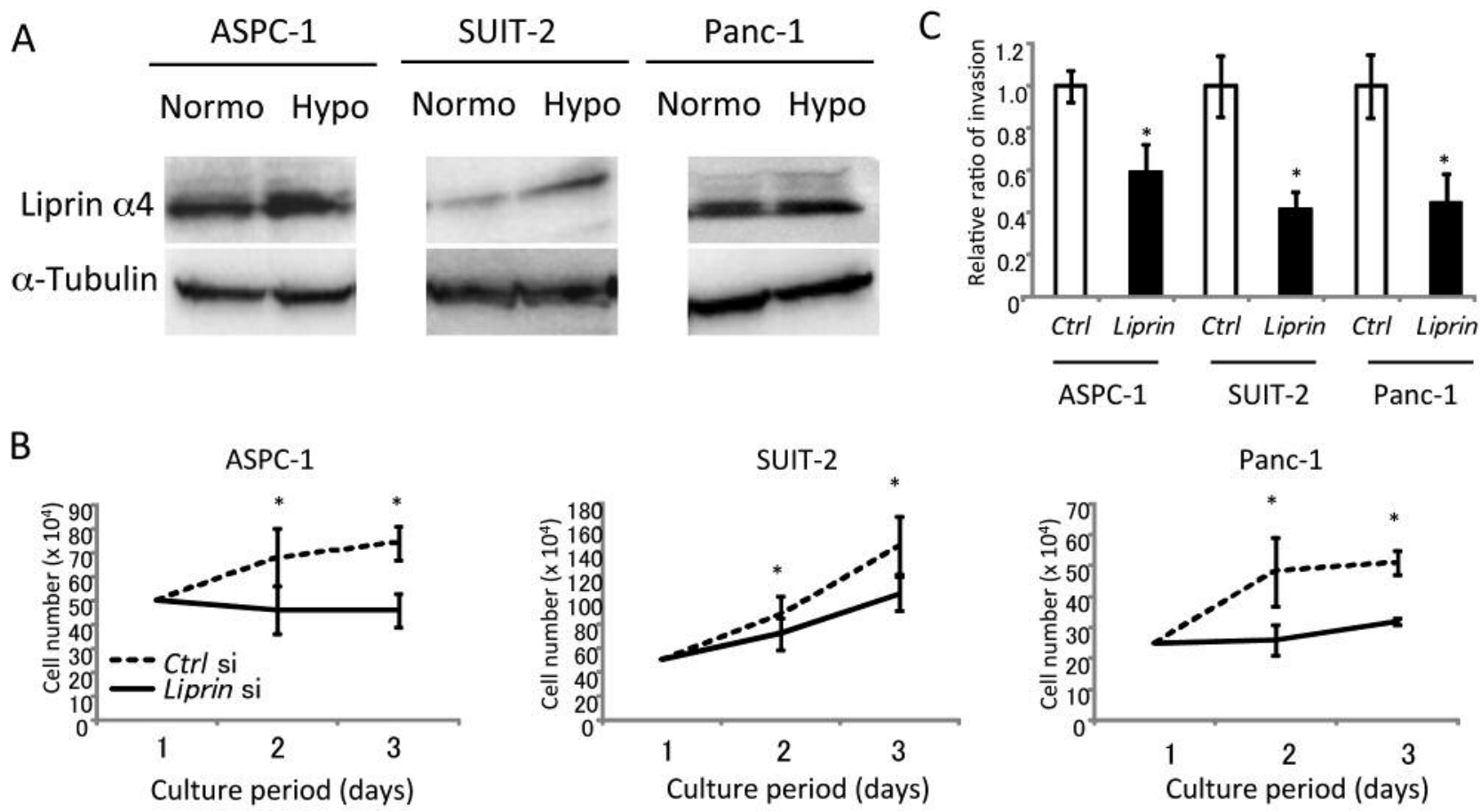

Figure 1. Hypoxia-induced liprin- $\alpha 4$ contributes to proliferation and invasiveness in pancreatic ductal adenocarcinoma (PDAC). A: Expression of liprin- $\alpha 4$ in PDAC cells under normoxia and hypoxia was estimated by western blot. B: Cell proliferation was analyzed. Cell numbers at the indicated days in PDAC cells transfected with liprin- $\alpha 4$ siRNA were counted under light microscopy. C: Cell invasiveness was estimated by matrigel invasion assay. All cells that had migrated from the upper to the lower side of the filter were counted. Data are presented as means $\pm S D$. *Significantly different at $p<0.05$. Ctrl: Transfected with control siRNA.

Statistical analysis. The data are presented as the means \pm standard deviation (SD). Student's $t$-tests were used to compare continuous variables between two groups. Differences with $p$-values of less than 0.05 were considered as statistically significant.

\section{Results}

Liprin- $\alpha 4$ contributes to proliferation and invasiveness of PDAC cells under hypoxia. Firstly, we analyzed the genes up-regulated under hypoxia in two PDAC cell lines in a microarray experiment. Because the increase of liprin- $\alpha 4$ gene was the highest, we selected liprin- $\alpha 4$ as a candidate gene for further study (Table I). We confirmed that liprin- $\alpha 4$ expression was increased at the protein level under hypoxia compared with normoxia (Figure 1A).

In order to ascertain the biological roles of liprin- $\alpha 4$, liprin$\alpha 4$ expression was knocked down using liprin- $\alpha 4$ siRNA. The number of proliferating cells in liprin- $\alpha 4$ siRNA-transfected PDAC cells were significantly lower than those in the control in all three PDAC cell lines under hypoxia (Figure 1B). Invasiveness of liprin- $\alpha 4$ siRNA-transfected PDAC cells was also significantly lower compared with the control in the three PDAC cell lines under hypoxia (Figure 1C). These results suggest that liprin- $\alpha 4$ is involved in proliferation and invasiveness of PDAC cells under hypoxic conditions.

Phosphoinositide 3-kinase (PI3K) and mitogen-activated protein kinase (MAPK) signaling pathways may contribute to liprin- $\alpha 4$-induced activation in PDAC cells. PI3K and MAPK pathways are thought to play a pivotal role in cell proliferation. Thus, we investigated whether PI3K and MAPK pathways contribute to liprin- $\alpha 4$-induced activation under hypoxia. Phospho AKT and phospho ERK decreased when liprin- $\alpha 4$ was inhibited in ASPC-1 cells and SUIT-2 cells, while there was no significant difference in Panc-1 cells (Figure 2). These results suggest that PI3K and MAPK pathways may play a role in liprin- $\alpha 4$-induced activation under hypoxia.

Endothelial-mesenchymal transition (EMT) is involved in liprin$\alpha 4$-induced invasiveness. EMT and MMP are thought to be important mechanisms in invasiveness. Of the MMP family, MMP2 and MMP9 are reported to play pivotal roles in invasiveness under hypoxia $(5,6)$. Therefore, the expressions of EMT-related molecules and MMP2/MMP9 were investigated. The expression of E-cadherin was increased and the expressions of vimentin, SNAIL, and TWIST were reduced in liprin- $\alpha 4$ 


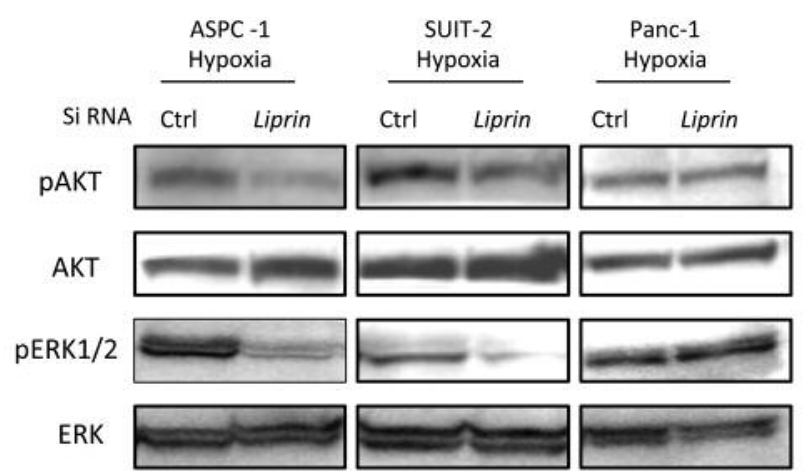

Figure 2. Phosphoinositide 3-kinase (PI3K) and mitogen-activated protein kinase $(M A P K)$ pathways contribute to liprin-a4-induced activation in pancreatic ductal adenocarcinoma (PDAC) cells. Expressions of pAKT, AKT, phospho-extracellular signal-regulated kinase (pERK) 1/2 and ERK1/2 under hypoxia in PDAC cells transfected with liprin- $\alpha 4$ siRNA were analyzed by western blot. Ctrl: Transfected with control siRNA.

siRNA-transfected PDAC cells (Figure 3). Meanwhile, liprin- $\alpha 4$ knockdown did not affect the expression of MMP2 and MMP9. These results suggest that liprin- $\alpha 4$ induces invasiveness under hypoxia in PDAC cells through EMT.

Liprin-a4 contributes to tumor progression in vivo. To evaluate the results of proliferation observed in vitro, $\mathrm{BALB} / \mathrm{c}$ nude mice $(\mathrm{n}=3)$ were subcutaneously implanted with SUIT-2 cells transfected with liprin- $\alpha 4$-targeting siRNA or non-targeting control siRNA. Tumor volumes in mice implanted with liprin- $\alpha 4$-transfected SUIT-2 cells were significantly lower than those in control mice (Figure 4A). However, tumors were formed in all mice in both groups, suggesting that there was no significant difference in tumorigenesis between the two groups. Next, in order to analyze this mechanism of reduced growth, we performed Ki67 and VEGF staining of tumors from mice, which showed that tumors from mice injected with SUIT-2 cells transfected with liprin- $\alpha 4$-siRNA had lower Ki67 and VEGF expressions than tumors from cells transfected with control siRNA (Figure 4B). Expressions of pAKT and pERK1/2 in liprin- $\alpha 4-$ siRNA-transfected tumors were also lower than those in the control tumors (Figure 4B). These results suggest that liprin$\alpha 4$ contributes to tumor progression and proliferation in vivo.

\section{Discussion}

Previously, we have demonstrated many important molecules or pathways that are activated under hypoxia and that contribute to the induction of a malignant phenotype in PDAC cells. For example, Hh signaling is activated under

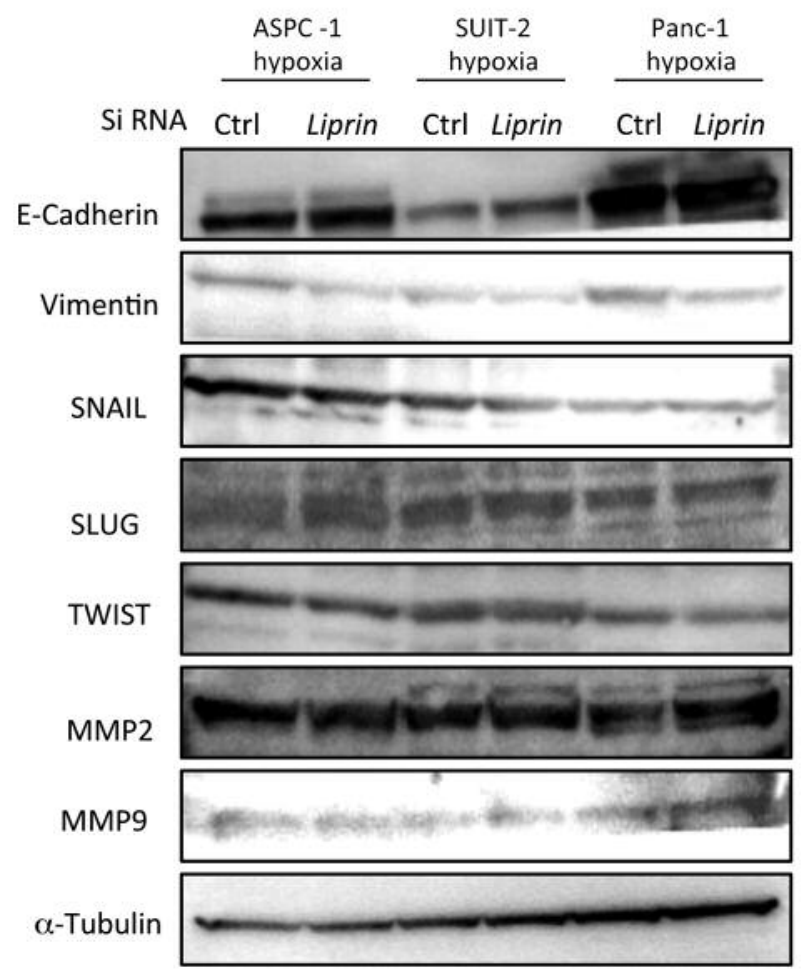

Figure 3. Endothelial-mesenchymal transition (EMT) is involved in liprin-a4-induced invasiveness of pancreatic ductal adenocarcinoma (PDAC) cells. Expressions of EMT-related molecules and matrix metalloproteinase (MMP) 2 and MMP9 under hypoxia in PDAC cells transfected with liprin- $\alpha 4$ siRNA were estimated by western blot. Ctrl: Transfected with control siRNA.

hypoxia through the up-regulation of MAML3 and RBPJ, and they play pivotal roles in hypoxia-induced proliferation, invasiveness, and tumorigenesis of pancreatic cancer $(5,6)$. In the present study, to detect a new molecule targeting pancreatic cancer under hypoxic conditions, we explored the results of microarray analysis in pancreatic cancer cells cultured under normoxia and hypoxia. We selected liprin- $\alpha 4$ and analyzed whether it contributes to the hypoxia-induced malignant phenotype in pancreatic cancer. Firstly, we expected that liprin- $\alpha 4$ would be related to Hh signaling activation under hypoxia. However, unexpectedly, liprin- $\alpha 4$ did not affect the expressions of GLI family zinc finger 1 (GLI1), which is a target gene of Hh signaling (data not shown). As shown in Figure 2, liprin- $\alpha 4$-induced proliferation may be induced through PI3K or MAPK signaling pathways under hypoxia. Liprin- $\alpha 4$-induced invasion may occur through EMT. However, Panc- 1 cells showed different kinetics of PI3K and MAPK signaling. This may be because tumors have many mutations in signal transduction, which may cause refractory pancreatic cancer. 


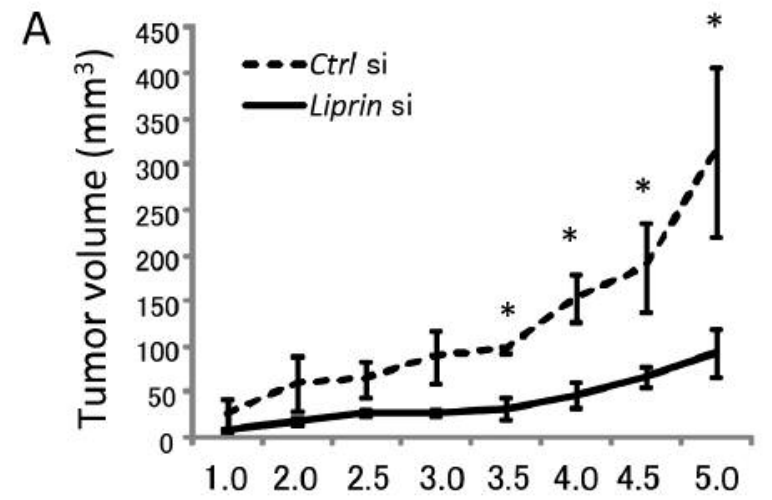

Time after tumor injection (week)

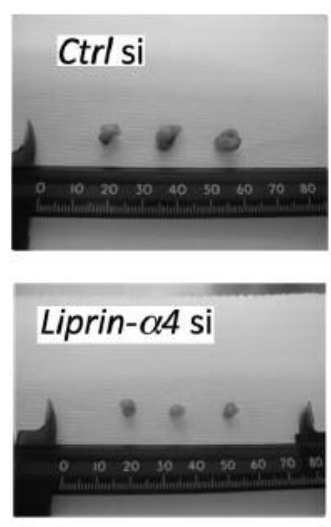

B

Ki67
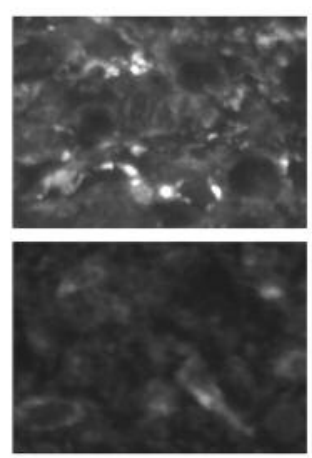

VEGF
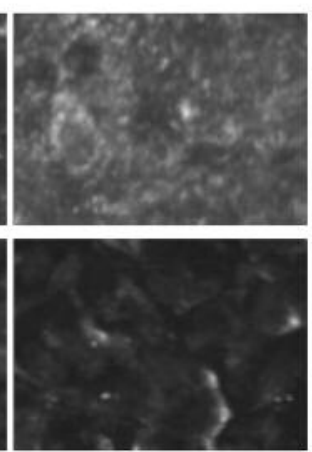

PAKT
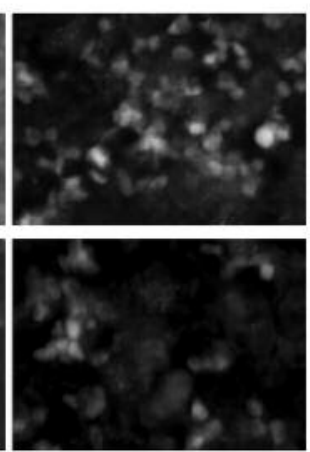

PERK1/2
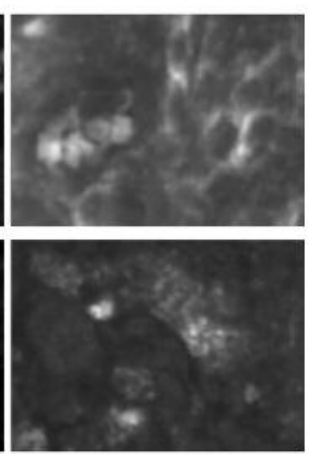

Figure 4. Liprin-a4 contributes to tumor progression in vivo. A: SUIT-2 cells transfected with liprin- $\alpha 4$-targeting siRNA or non-targeting control siRNA (Ctrl) were subcutaneously implanted into flank regions $\left(1.0 \times 10^{6}\right.$ cells in matrigel) of BALB/c nude mice $(n=3)$. The tumor size was determined on the indicated days. Data are presented as means $\pm S D$. *Significantly different at $p<0.05$. Representative photographs of mice (5 weeks after tumor injection) are shown. B: Immunofluorescence staining of Ki67, vascular endothelial growth factor (VEGF), pAKT and phosphoextracellular signal-regulated kinase ( $p E R K$ ) 1/2 were performed using tumors from mice. Original magnification is $\times 400$.

With respect to the extent of the decrease, liprin- $\alpha 4$ was likely to contribute to invasion more than proliferation. In fact, many research articles suggest that liprin is related to the invasive ability of cancer cells $(12,13)$. From our results, a relationship between liprin- $\alpha 4$ and E-cadherin is suggested. However, our results showing that the suppression of liprin- $\alpha 4$ leads to an increase in E-cadherin are opposite to those of Mattauch et al. (7). The difference in cancer types; renal cell carcinoma and PDAC may have caused this discrepancy. MMP2 and MMP9 were found to be unlikely to contribute to liprin- $\alpha 4$-induced invasion under hypoxia, while we have shown that they were involved in $\mathrm{Hh}$ signaling-induced invasiveness under hypoxia $(5,6)$. Taken together, our results demonstrate novel mechanisms of proliferation and invasion of pancreatic cancer cells under hypoxia, suggesting that liprin$\alpha 4$ may be a potential therapeutic target molecule.
There are still only a limited number of reports about the biological significance of liprin- $\alpha 4$ in cancer cells. Figure 5 depicts our hypothesis that liprin- $\alpha 4$ is involved with proliferation and invasion through PI3K and MAPK pathways in pancreatic cancer under hypoxic conditions. Because liprin- $\alpha 4$ is expressed more under hypoxia compared with normoxia, it is difficult to focus on liprin- $\alpha 4$ from the point of view of experiments performed under ordinary normoxic conditions. We believe that liprin- $\alpha 4$ could be a good therapeutic target for pancreatic cancer, whose microenvironment is extremely hypoxic. The cancer microenvironment now receives much attention. Therefore, we believe that our research focusing on the microenvironment of one cancer type under hypoxia is significantly important as an approach from an unusual point of view. Research into liprin expression induced under hypoxia may be useful for the development of a new effective therapeutic strategy against refractory pancreatic cancer. 


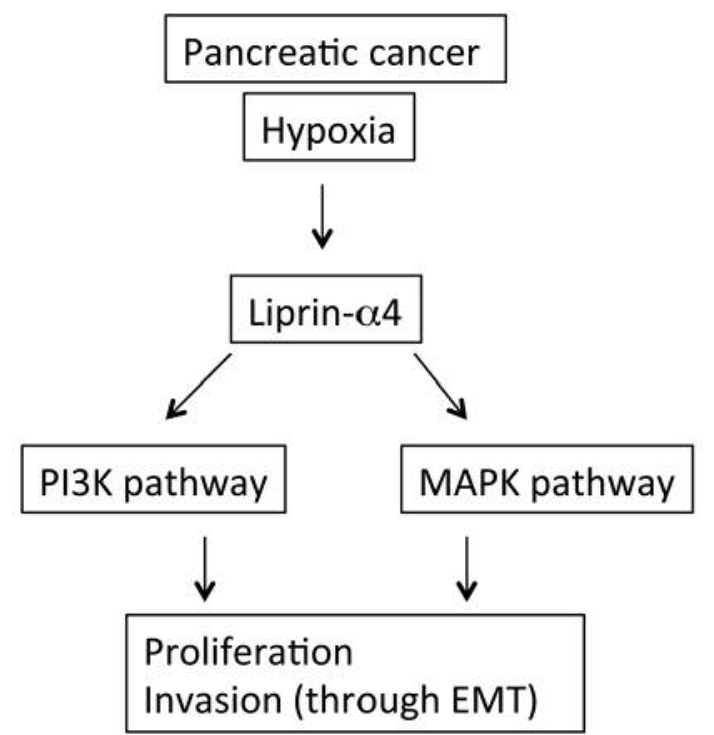

Figure 5. Model of our findings in this study. Liprin- $\alpha 4$ is involved in proliferation and invasion through phosphoinositide 3-kinase (PI3K) and mitogen-activated protein kinase (MAPK) pathways in pancreatic cancer under hypoxic conditions.

\section{Conflicts of Interest}

The Authors declare no financial or commercial conflict of interest in regard to this study.

\section{Acknowledgements}

The Authors thank Ms. Kaori Nomiyama and Ms. Emi Onishi for skillful technical assistance. This study was supported by JSPS KAKENHI Grant Number 17H04283.

\section{References}

1 Jemal A, Siegel R, Ward E, Hao Y, Xu J and Thun MJ: Cancer statistics 2009. CA Cancer J Clin 59: 225-249, 2009.

2 Koong AC, Mehta VK, Le QT, Fisher GA, Terris DJ, Brown JM, Bastidas AJ and Vierra M: Pancreatic tumors show high levels of hypoxia. Int J Radiat Oncol Biol Phys 48: 919-922, 2000.

3 Koukourakis MI, Giatromanolaki A, Skarlatos J, Corti L, Blandamura S, Piazza M, Gatter KC and Harris AL: Hypoxia inducible factor (HIF-1 $\alpha$ and HIF-2 $\alpha$ ) expression in early esophageal cancer and response to photodynamic therapy and radiotherapy. Cancer Res 61: 1830-1832, 2001.
4 Nakamura K, Martin KC, Jackson JK, Beppu K, Woo CW and Thiele CJ: Brain-derived neurotrophic factor activation of TrkB induces vascular endothelial growth factor expression via hypoxia-inducible factor-1alpha in neuroblastoma cells. Cancer Res 66: 4249-4255, 2006.

5 Onishi H, Kai M, Odate S, Iwasaki H, Morifuji Y, Ogino T, Morisaki T, Nakashima Y and Katano M: Hypoxia activates the hedgehog signaling pathway in a ligand-independent manner by up-regulation of $S M O$ transcription in pancreatic cancer. Cancer Sci 102: 1144-1150, 2011.

6 Onishi H, Yamasaki A, Kawamoto M, Imaizumi A and Katano M: Hypoxia but not normoxia promotes Smoothened transcription through up-regulation of RBPJ and Mastermindlike 3 in pancreatic cancer. Cancer Lett 371: 143-150, 2016.

7 Mattauch S, Sachs $M$ and Behrens J: Liprin- $\alpha 4$ is a new hypoxia-inducible target gene required for maintenance of cellcell contacts. Exp Cell Res 316: 2883-2392, 2010.

8 Serra-Pagès C, Medley QG, Tang M, Hart A and Streuli M: Liprins, a family of LAR transmembrane protein-tyrosine phosphataseinteracting proteins. J Biol Chem 273: 15611-15620, 1998.

9 Serra-Pagès C, Kedersha NL, Fazikas L, Medley Q, Debant A and Streuli M: The LAR transmembrane protein tyrosine phosphatase and a coiled-coil LAR-interacting protein colocalize at focal adhesions. EMBO J 14: 2827-2838, 1995.

10 Spangler SA and Hoogenraad CC: Liprin-alpha proteins: scaffold molecules for synapse maturation. Biochem Soc Trans 35: 1278-1282, 2007.

11 Shen JC, Unoki M, Ythier D, Duperray A, Varticovski L, Kumamoto K, Pedeux R and Harris CC: Inhibitor of growth 4 suppresses cell spreading and cell migration by interacting with a novel binding partner, liprin alpha1. Cancer Res 67: 2552-2558, 2007.

12 Astro V, Asperti C, Cangi MG, Doglioni C and de Curtis I: Liprin- $\alpha 1$ regulates breast cancer cell invasion by affecting cell motility, invadopodia and extracellular matrix degradation. Oncogene 30: 1841-1849, 2011.

13 Pehkonen H, von Nandelstadh P, Karhemo PR, Lepikhova T, Grenman R, Lehti $\mathrm{K}$ and Monni O: Liprin- $\alpha 1$ is a regulator of vimentin intermediate filament network in the cancer cell adhesion machinery. Sci Rep 14: 24486, 2016.

14 Suyama K, Onishi H, Imaizumi A, Shinkai K, Umebayashi M, Kubo M, Mizuuchi Y, Oda Y, Tanaka M, Nakamura M and Katano M: CD24 suppresses malignant phenotype by downregulation of $S H H$ transcription through STAT1 inhibition in breast cancer cells. Cancer Lett 374: 44-53, 2016.

15 Miyahara E, Nishikawa T, Takeuchi T, Yasuda K, Okamoto Y, Kawano Y and Horiuchi M: Effect of myeloperoxidase inhibition on gene expression profiles in HL-60 cells exposed to $1,2,4,-$ benzenetriol. Toxicology 317: 50-57, 2014.

Received September 20, 2017

Revised October 6, 2017

Accepted October 13, 2017 